\title{
3-N-Butyphthalide improves learning and memory in rats with vascular cognitive impairment by activating the SIRT1/BDNF pathway
}

\author{
AYONG TIAN ${ }^{1}$, WAN LI ${ }^{2}$, QING ZAI ${ }^{1}, \mathrm{HUI} \mathrm{LI}^{3}$ and RONG-WEI ZHANG ${ }^{3}$ \\ ${ }^{1}$ Department of Anesthesiology, The First Affiliated Hospital of China Medical University, Shenyang, Liaoning 110001; \\ ${ }^{2}$ Department of Neurology, The Ninth People's Hospital of Shenyang, Shenyang, Liaoning 110024; ${ }^{3}$ Department of \\ Gerontology and Geriatrics, The First Affiliated Hospital of China Medical University, \\ Shenyang, Liaoning 110001, P.R. China
}

Received July 3, 2019; Accepted April 4, 2020

DOI: $10.3892 / \mathrm{mmr} .2020 .11106$

\begin{abstract}
Vascular cognitive impairment (VCI) is a type of cerebral vascular disorder that leads to learning and memory decline. VCI models can be induced by chronic cerebral hypoperfusion via permanent bilateral common carotid artery occlusion. 3-N-Butylphthalide (NBP) is a neuroprotective drug used for the treatment of ischemic cerebrovascular diseases. Silent information regulator 1 (SIRT1) plays an important role in memory formation and cognitive performance, and its abnormal reduction is associated with cognitive dysfunction in neurodegenerative diseases. Brain-derived neurotrophic factor (BDNF) is a neurotrophic factor that plays critical roles in promoting neuronal growth and injury repair. The present study was performed to investigate the effects and the underlying mechanism of NBP on learning deficits in a rat model of VCI. Rats were divided into a control group, model group, low-NBP-dose group (30 mg/kg/day), high-NBP-dose group $(60 \mathrm{mg} / \mathrm{kg} / \mathrm{day})$, NBP + SIRT1 inhibitor group and NBP + BDNF inhibitor group. Rats were then subjected to Morris water maze and T-maze tests, which identified that NBP treatment significantly attenuated memory impairments in VCI rats. Molecular examination indicated that SIRT1 and BDNF expression levels in the hippocampus were increased by NBP treatment. However, NBP failed to ameliorate cognitive function after inhibition of the SIRT1/BDNF signaling pathway. In addition, NBP in combination with a SIRT1 inhibitor suppressed BDNF protein expression, but inhibition of BDNF did not inhibit SIRT1 protein expression in rats with VCI. The present
\end{abstract}

Correspondence to: Professor Rong-Wei Zhang, Department of Gerontology and Geriatrics, The First Affiliated Hospital of China Medical University, 155 Nanjing North Street, Shenyang, Liaoning 110001, P.R. China

E-mail: rongweizhang@126.com

Key words: 3-N-butylphthalide, vascular cognitive impairment, silent information regulator 1 , brain-derived neurotrophic factor results suggested that the neuroprotective effects of NBP on learning deficits in a rat model of VCI may be via regulation of the SIRT1/BDNF signaling pathway, in which SIRT1 may be the upstream signaling molecule. Therefore, the SIRT1/BDNF pathway could be a potential therapeutic target for VCI.

\section{Introduction}

Vascular cognitive impairment (VCI) comprises a spectrum of clinical syndromes ranging from mild cognitive impairment to dementia, and is caused by cerebrovascular risk factors, such as hypertension, diabetes and smoking, and cerebrovascular diseases (1-3). VCI is likely to become the primary type of cognitive impairment in developing countries, and thus is becoming a serious social health problem (4). Although the molecular pathogenesis of VCI is unclear, current studies suggest that 3-N-butylphthalide (NBP), one of the chemical constituents in celery oil, improves VCI, thus new therapeutic targets are likely to be identified $(5,6)$.

Silent information regulator 1 (SIRT1) is a member of the sirtuin family that undergoes $\mathrm{NAD}^{+}$-dependent acetylation, and acts as a regulatory protein associated with aging and age-related degenerative diseases (7). SIRT1 plays an important role in a number of physiological and pathological processes, including senescence and metabolic regulation (8-10). SIRT1 is also involved in the regulation of endothelial cell gene expression after angiogenesis, suggesting that it may play an important protective role in chronic cerebral ischemia (11).

Brain-derived neurotrophic factor (BDNF) is a major neuroprotective factor with anti-apoptotic properties, which protects and promotes nerve regeneration (12). BDNF exerts its biological function by binding to its specific receptor, tropomyosin receptor kinase B (TrkB) (12). High expression levels of BDNF and TrkB are present in the hippocampus and cortex of the brain $(13,14)$. BDNF is the most effective biologically active substance for protecting neurons and regulating synaptic plasticity (15). However, expression levels of BDNF in hippocampal neurons are significantly decreased by hypoxia, suggesting that BDNF may play an important role in the pathogenesis of VCI (16). 
NBP is a novel drug for the treatment of ischemic cerebral injury that has been independently developed and researched in China (17). Several studies have reported that NBP reduces focal cerebral infarction volume in rats, improves brain energy metabolism disorders and reduces apoptotic neuronal cell death (18-20). NBP is a multi-target drug with several mechanisms, and thus has a variety of neuroprotective effects in brain tissues (21-23). The present study hypothesized that the regulation of the SIRT1/BDNF signaling pathway may be involved in the underlying mechanism of action of NBP. Therefore, the present study investigated the neuroprotective effects of NBP on learning and memory in a rat model of VCI induced by two-vessel occlusion, and also examined the role of the SIRT1/BDNF signaling pathway.

\section{Materials and methods}

Animals and groups. In total, 60 specific pathogen-free (SPF) Sprague-Dawley rats, (male; 2 months old; weight, 250-280 g) were purchased from Liaoning Longevity Biotechnology Co., Ltd. (Liao 2010-0201). Rats were housed in a SPF animal experiment room at $24 \pm 2^{\circ} \mathrm{C}$ with $40-70 \%$ humidity under a $12 \mathrm{~h}$ light/dark cycle, and were allowed free access to water and food. After 10 days of acclimatization, the rats were divided randomly into six groups: i) Sham operated control group (C group; $n=10)$; ii) model group (M group; $\mathrm{n}=10$ ); iii) NBP-low-dose group (L-NBP group; $30 \mathrm{mg} / \mathrm{kg} / \mathrm{day} ; \mathrm{n}=10$ ); iv) NBP-high-dose group (H-NBP group; $60 \mathrm{mg} / \mathrm{kg} / \mathrm{day} ; \mathrm{n}=10)$; v) SIRT1 inhibitor + NBP group (S+N group; NBP $60 \mathrm{mg} / \mathrm{kg} / \mathrm{day} ; \mathrm{n}=10)$; and vi) $\mathrm{BDNF}$ inhibitor + NBP group $(\mathrm{B}+\mathrm{N}$ group; NBP $60 \mathrm{mg} / \mathrm{kg} / \mathrm{day}$; $\mathrm{n}=10)$. Experiments were approved by The China Medical University Animal Care and Use Committee, and adhered to The Chinese Academy of Science Guidelines for Care and Use of Laboratory Animals.

Surgical procedure. The TrkB antagonist K-252 $\alpha$ (EMD Millipore) and sirtinol (Sigma-Aldrich; Merck KGaA) were used to inhibit BDNF and SIRT1, respectively. Rats were anesthetized by intraperitoneal injection of $10 \%$ chloral hydrate $(300 \mathrm{mg} / \mathrm{kg})$ and were then placed in the prone position on a rat brain stereotaxic device. Successful anesthesia was indicated when slow breathing, limb paralysis, dull pain reflex and loss of righting reflex occurred; individual rats showed mild abdominal distension but no signs of peritonitis after anesthesia. The catheter-insertion point was marked using the fontanelle as the zero point, Bregma after $1 \mathrm{~mm}$ and then right $1.5 \mathrm{~mm}$. A lateral ventricle catheter was inserted vertically to a depth of 3.5-3.8 mm 1 day before model induction surgery. Bilateral common carotid artery ligation is the most common method of inducing chronic cerebral ischemia in rats and has been widely used in VCI research $(24,25)$. The rats were weighed and injected intraperitoneally with $10 \%$ chloral hydrate $(300 \mathrm{mg} / \mathrm{kg})$ for anesthesia. A $\sim 2 \mathrm{~cm}$ incision was made in the skin and subcutaneous tissue around the midline of the neck, and the muscles were parted to expose the common carotid arteries. The common carotid arteries were peeled away, avoiding the vagus nerve and the bilateral common carotid arteries were then ligated.
Experimental design. The detailed protocol is shown in Fig. 1. Rats in the $\mathrm{S}+\mathrm{N}$ and $\mathrm{B}+\mathrm{N}$ groups underwent lateral ventricle catheterization and the experimental reagents were injected slowly into the lateral ventricle, leaving the needle in for 2-3 min. Rats in the $\mathrm{B}+\mathrm{N}$ group were administered $1 \mu \mathrm{g}$ /day $\mathrm{K}-252 \alpha$ and rats in the $\mathrm{S}+\mathrm{N}$ group were administered $1 \mathrm{mg} / \mathrm{kg}$ body weight/day sirtinol for 9 days. In addition to the $\mathrm{C}$ group, all rats in each group underwent artery ligation. Rats in the $C$ and $\mathrm{M}$ groups were fed with vegetable oil $\left(1 \mathrm{ml} / 100 \mathrm{~g}^{-1}\right)$ as a placebo. Rats in the H-NBP group and the two inhibitor groups were given NBP $60 \mathrm{mg} / \mathrm{kg} / \mathrm{day}$, and rats in the L-NBP group received NBP $30 \mathrm{mg} / \mathrm{kg} /$ day. NBP (dissolved in vegetable oil) or vegetable oil alone was administered via oral gavage once a day after bilateral common carotid artery occlusion (BCCAO). The NBP/vegetable oil treatments were administered for 28 consecutive days. During the experiment, the rats were weighed every week. NBP soft capsules were purchased from Shijiazhuang Pharmaceutical Co., Ltd. Then, 4 weeks after the surgery there were 10 rats in the $\mathrm{C}$ group, 9 in the $\mathrm{M}$ group, 9 in the H-NBP group, 9 in the L-NBP group, 8 in the $\mathrm{S}+\mathrm{N}$ group and 8 in the $\mathrm{B}+\mathrm{N}$ group.

T-maze test. T-maze tests can be used to assess spatial working memory (26). In the present study, rats were subjected to T-maze tests after 4 weeks of NBP treatment. Rats were maintained on a restricted feeding schedule at $85 \%$ of their free-feeding weight, and were habituated to the maze and accustomed to food rewards (a small amount of sugar). Each trial consisted of a sample run and a choice run. On the sample run, rats had to enter either the left or right arm to get the reward, while the other arm was blocked by a sliding door. On the choice run, the blocked door was removed and the rats were allowed to choose either arm freely. An interval of $10 \mathrm{sec}$ was allowed between the sample and choice runs. If the rats entered the previously unvisited arm, they were rewarded. The delayed alternation was then prolonged to 90 and $180 \mathrm{sec}$. Each daily session consisted of five trials, and each rat ran one trial at a time with an inter-trial interval of $10 \mathrm{~min}$. The number of corrections made by the rats when they entered the unvisited arm of the T-maze was measured.

Morris water maze (MWM) test. The MWM is designed to test spatial learning in rodents (27). Rats were trained twice a day for 5 consecutive days with an interval of $3 \mathrm{~h}$, and each trial lasted for $90 \mathrm{sec}$. The rats started from a different quadrant for each trial and the time to reach the platform (escape latency) was recorded using a video camera. The digital images were analyzed using water maze software (HVS Image 2020; HVS Image Software Ltd.). Additional probe trials were conducted, with the platform removed on the 6th day of the test. Swimming speed, times of crossing the platform, time spent in the quadrant previously containing the submerged platform and swimming distance were also recorded and represented as an index of memory.

Nissl staining. Rats were deeply anesthetized by intraperitoneal injection of sodium pentobarbital $(200 \mathrm{mg} / \mathrm{kg})$. When continuous spontaneous breathing had arrested for 2-3 min and muscle relaxation had occurred, the rats were perfused transcardially with $0.9 \%$ normal saline followed by $4 \%$ 
NBP

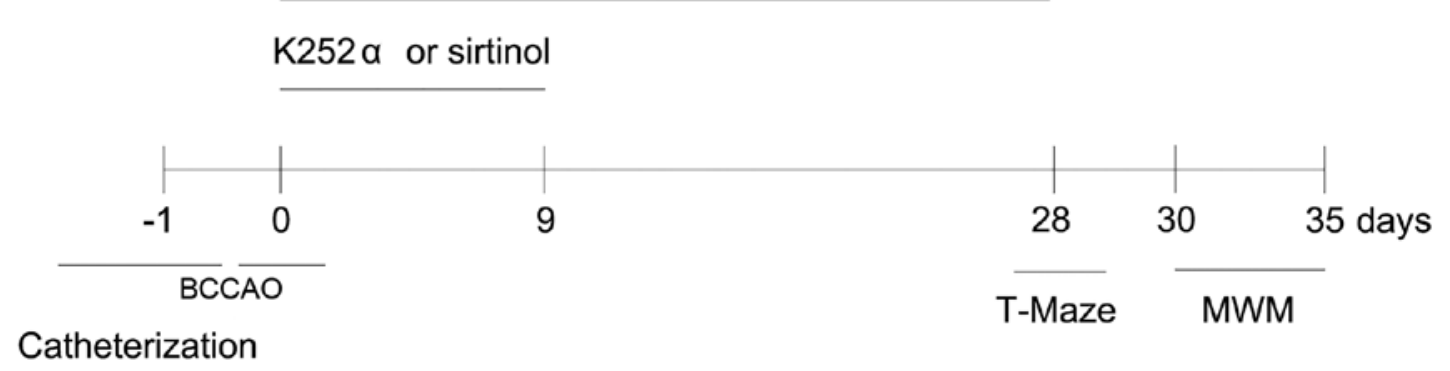

Figure 1. Timeline for experiments. One day prior to the operation, rats in the $\mathrm{S}+\mathrm{N}$ and $\mathrm{B}+\mathrm{N}$ groups underwent lateral ventricle catheterization. With the exception of the $\mathrm{C}$ group, all rats underwent vessel ligation, $24 \mathrm{~h}$ post-lateral ventricle catheterization. Rats in the $\mathrm{S}+\mathrm{N}$ and $\mathrm{B}+\mathrm{N}$ groups were administered $\mathrm{K}-252 \alpha$ and sirtinol by lateral ventricle for consecutive 9 days, and were given NBP via oral gavage for 28 consecutive days, other groups were also fed with vegetable oil or NBP via oral gavage for 28 consecutive days. T-maze tests were performed on day 28 and MWM tests were conducted on day 30 post-operation. Nissl staining, western blot analysis, immunofluorescence was performed on day 35. NBP, 3-N-Butylphthalide; BCCAO, bilateral common carotid artery occlusion; MWM, Morris water maze; S+N, SIRT1 inhibitor + NBP (60 mg/kg) group; B+N, BDNF inhibitor + NBP (60 mg/kg); C, control; M, model group.

paraformaldehyde in $0.1 \mathrm{M}$ sodium phosphate buffer ( $\mathrm{pH} 7.3)$. The brains were removed and immersed in $10 \%$ paraformaldehyde in sodium phosphate buffer for $24 \mathrm{~h}$ at $4^{\circ} \mathrm{C}$ for post-fixation, and then embedded in a single paraffin block. Sections were cut at $5 \mu \mathrm{m}$ and stained with $1 \%$ toluidine blue at $60^{\circ} \mathrm{C}$ for $40 \mathrm{~min}$, and Nissl-positive cells in the hippocampus were examined using a light microscope at $\mathrm{x} 400$ magnification (model BX53; Olympus Corporation) by two independent investigators who were blinded to the experimental conditions.

Immunofluorescence. Hippocampal tissues were removed and post-fixed in $4 \%$ paraformaldehyde ( $\mathrm{pH} 7.4$ ) at $4^{\circ} \mathrm{C}$ for $12 \mathrm{~h}$, and then cut at a thickness of $30 \mu \mathrm{m}$ using a vibrating microtome (Leica VT1000 S; Leica Biosystem GmbH). After blocking endogenous peroxidases and nonspecific binding sites with 10\% normal rabbit serum (cat. no. ab166640; Abcam) for $1 \mathrm{~h}$ at room temperature, the sections were incubated with primary rabbit anti-SIRT1 (1:800; cat. no. ab12193; Abcam) and rabbit anti-BDNF (1:800; cat. no. ab108319; Abcam) antibodies overnight at $4{ }^{\circ} \mathrm{C}$, followed by incubation with FITC-conjugated goat anti rabbit IgG (1:1,000; cat. no. ab6717; Abcam) and Cy3-conjugated goat anti-rabbit IgG (1:1,000; cat. no. ab6939; Abcam) at room temperature for $1 \mathrm{~h}$. After staining with DAPI (Sigma-Aldrich; Merck KGaA) for $10 \mathrm{~min}$ at $37^{\circ} \mathrm{C}$, the slides were examined under a fluorescence microscope (Eclipse E800; Nikon Corporation) at magnification x200, with a SPOT advanced digital camera (Diagnostic Instruments, Inc.). Fluorescent images were merged using Andor IQ version 2.0 software (Andor Technology Ltd.).

Western blotting. Rats were euthanized with an intraperitoneal injection of sodium pentobarbital $(200 \mathrm{mg} / \mathrm{kg})$ and sacrificed on day 28 after the final administration of NBP. Hippocampal tissues were dissected from rats and transferred into liquid nitrogen. For protein extraction, isolated tissue was homogenized in RIPA lysis buffer (cat. no. P0013B; Beyotime Institute of Biotechnology) on ice for $1 \mathrm{~h}$ and centrifuged at $12,000 \mathrm{x} \mathrm{g}$ for $10 \mathrm{~min}$ at $4^{\circ} \mathrm{C}$. The supernatant was collected and the protein concentration was quantified using a bicinchoninic acid protein assay kit (Sigma-Aldrich; Merck KGaA). Proteins (30 $\mu \mathrm{g} / \mathrm{lane}$ ) were loaded on a $10 \%$ gel, resolved using SDS-PAGE and subsequently transferred onto PVDF membranes (EMD Millipore).
The membranes were probed with primary antibodies against SIRT1 (1:1,000; cat. no. sc74465) and BDNF (1:1,000; cat. no. sc65514) overnight at $4{ }^{\circ} \mathrm{C}$, followed by incubation with a horseradish peroxidase-labeled secondary antibody $(1: 1,500$; cat. no. sc51625) at room temperature for $3 \mathrm{~h}$ (all purchased from Santa Cruz Biotechnology, Inc.). The proteins were then visualized by enhanced chemiluminescence (GE Healthcare) according to the manufacturer's instructions. The densities of the protein bands were analyzed quantitatively using Quantity-One software version 4.6.3 (Bio-Rad Laboratories, Inc.). GAPDH (1:5,000; cat. no. ab9485; Abcam) was used as a protein-loading control.

Statistical analysis. Data are presented as the mean \pm SD and were analyzed using SPSS 16.0 statistical software (SPSS, Inc.). Statistical significance was determined by one-way ANOVA followed by Tukey's test. $\mathrm{P}<0.05$ was considered to indicate a statistically significant difference.

\section{Results}

NBP reverses spatial working memory impairment in rats with $V C I$ in the T-maze test. The present results suggested that rats with VCI (M group) showed significantly reduced spontaneous alternation behavior compared with the control group $(\mathrm{P}<0.05)$. In addition, NBP (30 or $60 \mathrm{mg} / \mathrm{kg}$ ) significantly attenuated the impairment of spontaneous alternation behavior in rats with VCI $(\mathrm{P}<0.05)$, including when the interval between the sample and choice run was delayed by 90 or $180 \mathrm{sec}(\mathrm{P}<0.05)$. Therefore, the present results suggested that NBP improved working memory deficits in VCI rats (Fig. 2A).

NBP reverses learning and memory deficits in rats with VCI in the MWM test. The escape latencies in the different groups are shown in Fig. 2B. There was a significant difference in training test performance among the four different groups from day 2 , VCI rats ( $\mathrm{M}$ group) took longer to find the platform compared with the controls $(\mathrm{P}<0.05)$, while treatment with NBP $(30$ or $60 \mathrm{mg} / \mathrm{kg}$ ) decreased the escape latency compared with the VCI rats $(\mathrm{P}<0.05$; Fig. 2B). In the probe test, the time spent and the swimming distance in the target quadrant, as well as the number of platform crossings were all significantly lower in 

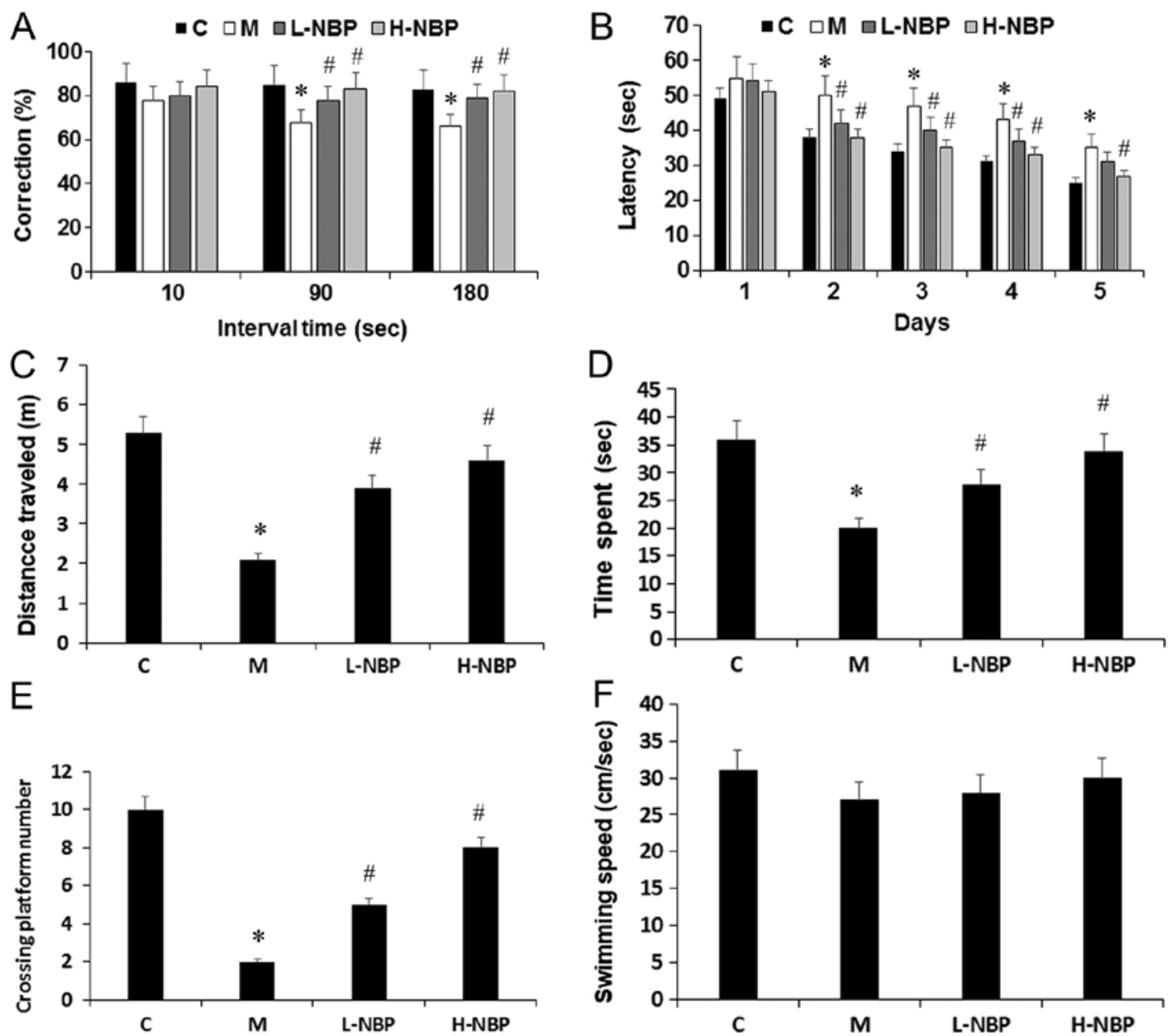

Figure 2. Protective effects of NBP against ischemic injury-induced spatial memory impairment in T-maze and MWM test. (A) Correction alternation was examined using a T-maze and tested using various intervals at 10,90 and $180 \mathrm{sec}$ delay. (B) Latency to reach the platform during the training period in MWM test. (C) Distance travelled, (D) time spent in the target quadrant and (E) the crossing platform numbers during the probe trail in the MWM test. (F) Swimming speed among the groups. Data are presented as the mean $\pm \mathrm{SD}$. C, $\mathrm{n}=10 ; \mathrm{M}, \mathrm{n}=9 ; \mathrm{L}-\mathrm{NBP}, \mathrm{n}=9 ; \mathrm{H}-\mathrm{NBP}, \mathrm{n}=9 .{ }^{*} \mathrm{P}<0.05$ vs. C group. ${ }^{*} \mathrm{P}<0.05$ vs. M group. NBP, 3-N-butylphthalide; C, control; M, model group; L-NBP, low dose NBP group (30 mg/kg); H-NBP, high dose NBP group (60 mg/kg); MWM, Morris water maze.

the $\mathrm{M}$ group compared with the control group $(\mathrm{P}<0.05)$. NBP $(30 \mathrm{or} 60 \mathrm{mg} / \mathrm{kg}$ ) increased the time spent and the swimming distance in the target quadrant, and improved the search performance as indicated by a higher number of platform crossings compared with the $\mathrm{M}$ group $(\mathrm{P}<0.05$; Fig. $2 \mathrm{C}$-E). However, swimming speed was similar in all groups during the trials (Fig. 2F). Collectively, the present results suggested that NBP may improve learning and memory impairments in VCI rats.

$N B P$ reversal of cognitive deficits in rats with VCI may be related to SIRT1 signaling. To examine the role of SIRT1 signaling in the neuroprotective effect of NBP against VCI rats, the present study investigated the cognitive performance of rats treated with NBP + SIRT1 antagonist (S+N) using the MWM test. The present results suggested that NBP reversed learning and memory deficits in rats with VCI in the MWM test, as indicated by shorter escape latencies, longer swimming distance and increased platform crossings (Fig. 2). However, the escape latencies of the VCI rats significantly increased after treatment with NBP + SIRT1 antagonist $(\mathrm{S}+\mathrm{N})$, the swimming distance was significantly shorter and the number of platform crossings decreased compared with the NBP group $(\mathrm{P}<0.05$; Fig. 3$)$. Therefore, the present results suggested that SIRT1 antagonists may partially inhibit the spatial memory and learning improvements caused by NBP. However, this inhibition was not significant in the NBP+BDNF antagonist treatment $(\mathrm{B}+\mathrm{N})$ group (Fig. 3).

NBP suppresses neuronal loss in the hippocampal cornu Ammonis 1 (CAl) region in VCI rats. The present results suggested that there were no histopathologic abnormalities in the hippocampus in control rats (Fig. 4). However, VCI rats showed significant neuronal loss and neuronal structural degeneration in the CA1 region, while these effects were attenuated by $60 \mathrm{mg} / \mathrm{kg}$ NBP (H-NBP), as reflected by the density and morphology of neurons with Nissl bodies $(\mathrm{P}<0.05)$. Collectively, the present results suggested that NBP may inhibit hippocampal neuronal death in VCI rats.

NBP upregulates SIRTI and BDNF protein expression levels in the hippocampus of VCI rats, which is reversed by SIRTI inhibition. The present study investigated the effects of NBP on SIRT1 and BDNF expression levels using immunofluorescence. SIRT1-positive and BDNF-positive cells were lacking in the VCI group (M group) compared with the control group (C group). However, the expression levels of these proteins was increased by $60 \mathrm{mg} / \mathrm{kg}$ NBP treatment (H-NBP group) 

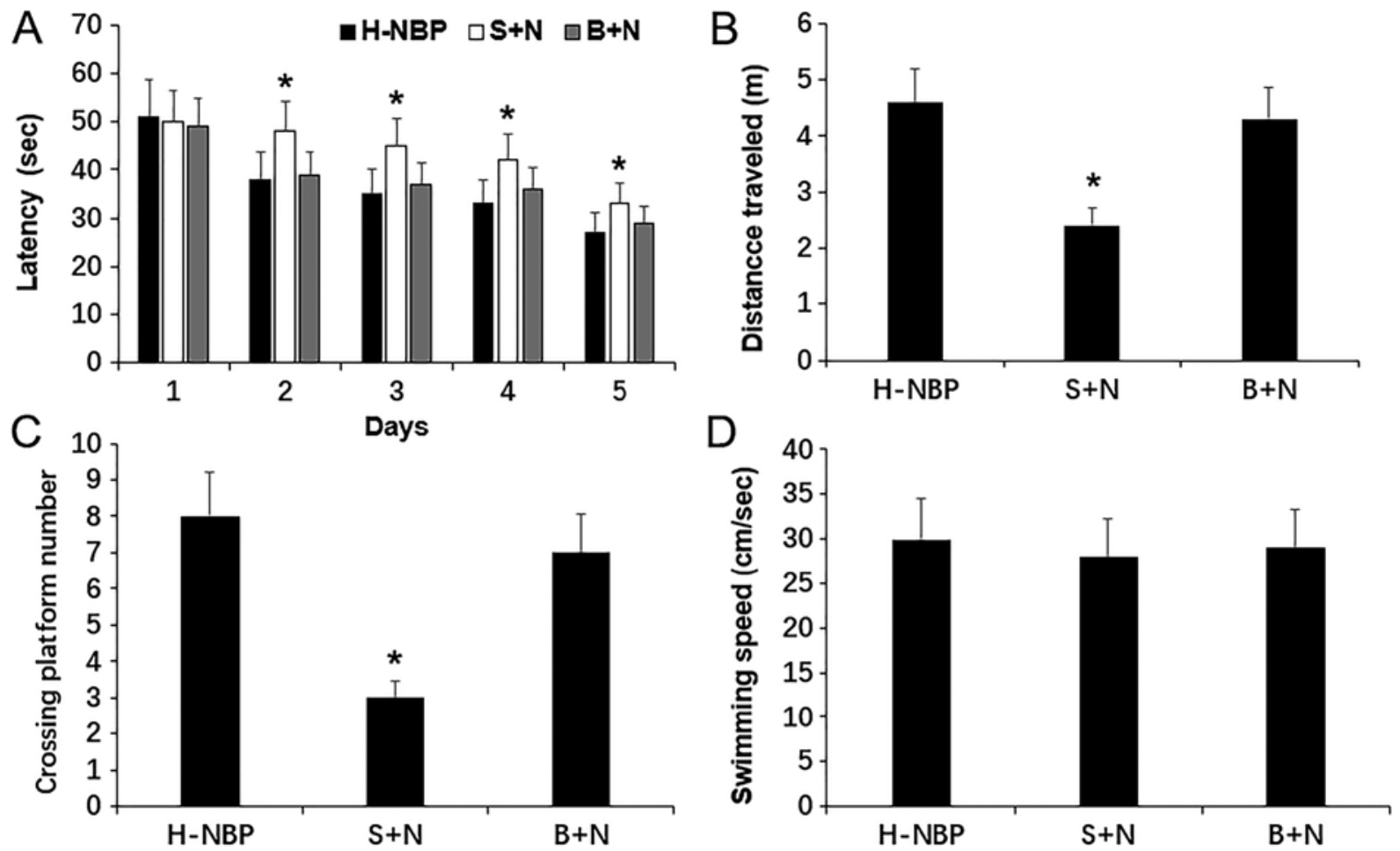

Figure 3. Cognitive performance of NBP in vascular cognitive impaired rats after the blockade of SIRT1 measured by MWM test. (A) Latency to reach the platform during the training period in MWM test. (B) Representative swimming distance. (C) Crossing platform numbers in the probe trail. (D) Swimming speed among the groups. Data are presented as the mean $\pm \mathrm{SD}$. H-NBP, $\mathrm{n}=9 ; \mathrm{S}+\mathrm{N}, \mathrm{n}=8 ; \mathrm{B}+\mathrm{N}, \mathrm{n}=8$. ${ }^{*} \mathrm{P}<0.05$ vs. H-NBP group. NBP, 3-N-butylphthalide; BDNF, brain-derived neurotrophic factor; SIRT1, silent information regulator 1; H-NBP, high dose NBP group (60 mg/kg); MWM, Morris water maze; S+N, SIRT1 inhibitor + NBP $(60 \mathrm{mg} / \mathrm{kg})$ group; B+N, BDNF inhibitor + NBP $(60 \mathrm{mg} / \mathrm{kg})$.
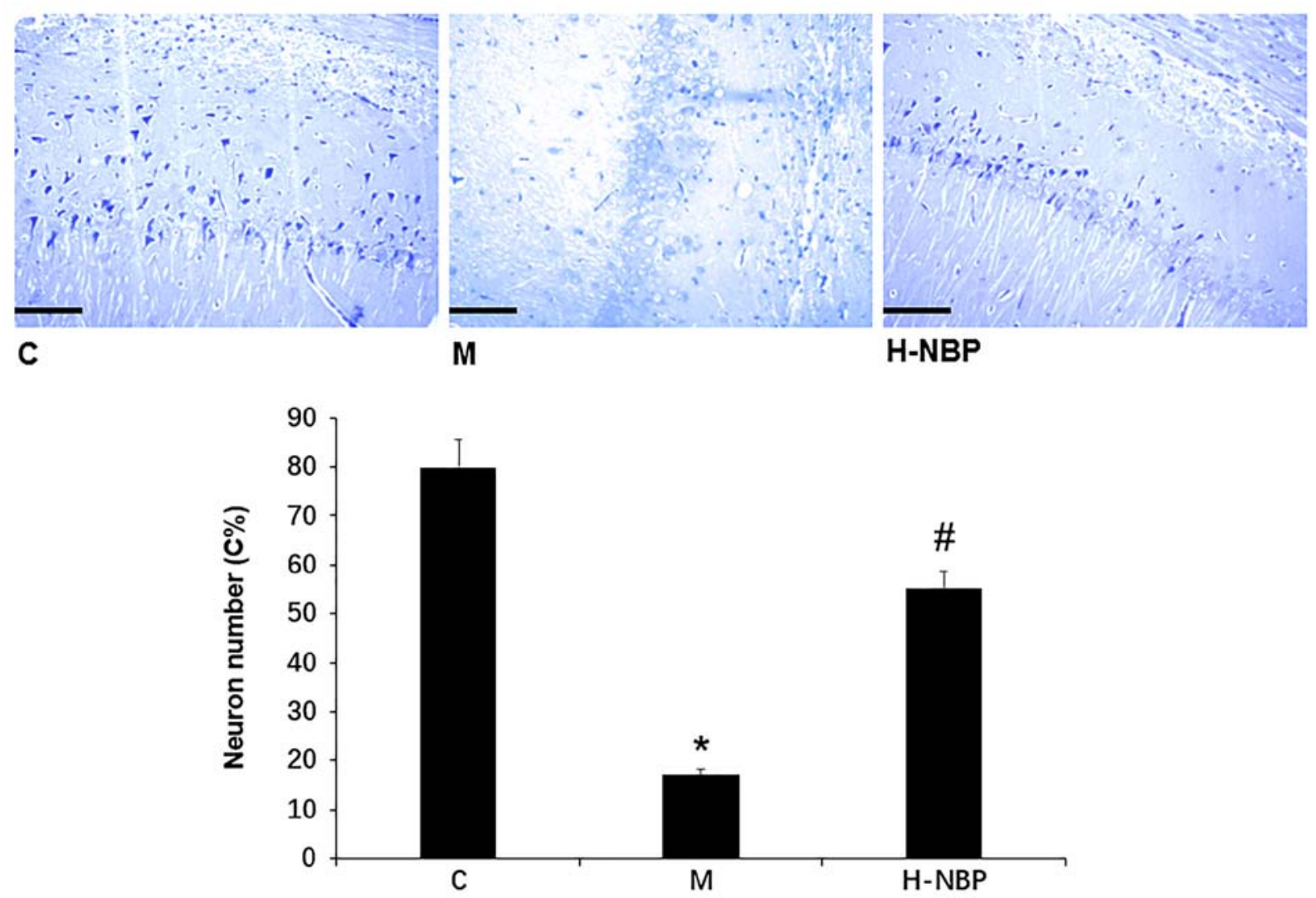

Figure 4. Hippocampal neuronal survival in CA1 region observed by Nissl staining. NBP attenuated ischemic injury-induced neuron loss in the CA1 region, as reflected by the number of the Nissl bodies. Magnification, x400; scale bar, $20 \mu \mathrm{m}$. Relative number of neurons with Nissl bodies of different groups was quantified. Data are presented as the mean $\pm \mathrm{SD}$. "P<0.05 vs. C group. "P<0.05 vs. M group. NBP, 3-N-butylphthalide; C, control; M, model group; H-NBP, high dose NBP group (60 mg/kg); CA1, cornu Ammonis 1. 

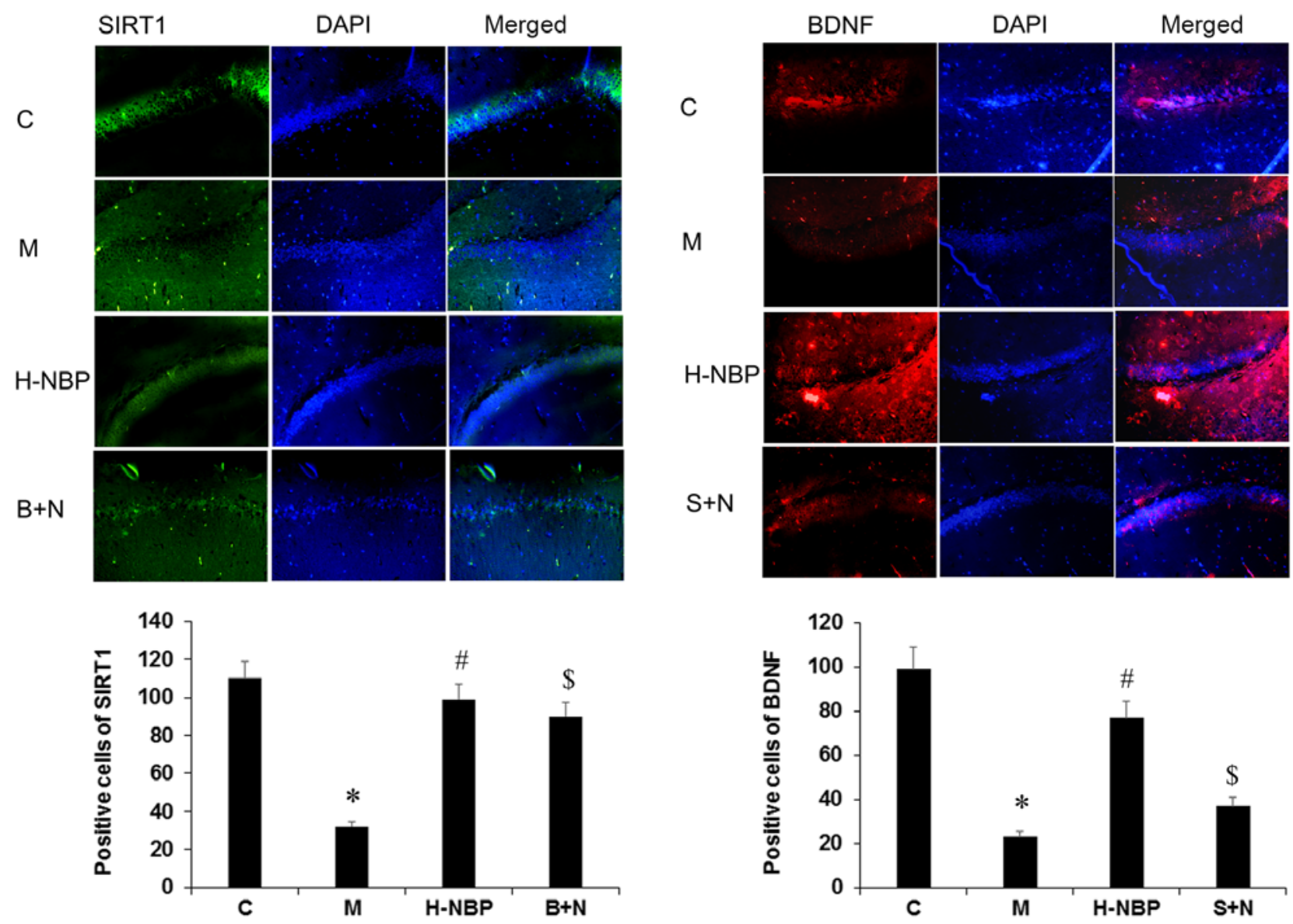

Figure 5. Effects of NBP on the expression levels of SIRT1 and BDNF in the hippocampus of VCI rats by immunofluorescence analysis. Magnification, x400. The intensity of the red or green fluorescence signal represents the expression level of the corresponding protein. The nucleus was counterstained with DAPI, which is represented by blue fluorescence. NBP reversed the decrease of SIRT1 and BDNF expression levels in VCI rats, as reflected by the positive labeled cells. BDNF expression level was reduced in $\mathrm{S}+\mathrm{N}$ group, but SIRT1 expression level was not decreased in $\mathrm{B}+\mathrm{N}$ group, compared with $\mathrm{H}-\mathrm{NBP}$ group. Data are presented as the mean $\pm \mathrm{SD}$. ${ }^{*} \mathrm{P}<0.05$ vs. C group. ${ }^{*} \mathrm{P}<0.05$ vs. M group. ${ }^{\$} \mathrm{P}<0.05$ vs. H-NBP group. NBP, 3-N-butylphthalide; BDNF, brain-derived neurotrophic factor; SIRT1, silent information regulator 1; H-NBP, high dose NBP group $(60 \mathrm{mg} / \mathrm{kg}) ; \mathrm{S}+\mathrm{N}$, SIRT1 inhibitor + NBP $(60 \mathrm{mg} / \mathrm{kg})$ group; B+N, BDNF inhibitor + NBP (60 mg/kg); C, control; M, model group; VCI, vascular cognitive impairment.

compared with the VCI group $(\mathrm{P}<0.05$; Fig. 5). The present results suggested that BDNF-positive cells were significantly reduced by SIRT1 inhibition ( $\mathrm{S}+\mathrm{N}$ group) compared with the H-NBP group $(\mathrm{P}<0.05)$, but SIRT1-positive cells were not reduced by BDNF inhibition.

The present study also investigated the effect of NBP on the expression levels of SIRT1 and BDNF by western blotting. SIRT1 and BDNF protein expression levels were reduced in the VCI rats ( $\mathrm{M}$ group) compared with control (C group) rats, and this effect was reversed by NBP treatment $(\mathrm{P}<0.05$; Fig. 6$)$. In addition, compared with the H-NBP group, the use of a SIRT1 inhibitor significantly decreased BDNF protein expression level in the hippocampus of VCI rats $(\mathrm{P}<0.05)$, but use of a BDNF inhibitor had no effect on SIRT1 protein expression compared with the H-NBP group. Therefore, the present results indicated that NBP increased SIRT1 and BDNF protein expression levels in the hippocampus of VCI rats, and that SIRT1 may be the upstream signaling factor in the SIRT1/BDNF pathway.

\section{Discussion}

Vascular cognitive impairment (VCI) has been the focus of noteworthy scientific research. VCI is a brain dysfunction syndrome, mainly caused by cerebrovascular disease $(28,29)$, consisting of a progression from mild cognitive impairment to dementia (30). However, VCI is not a single disorder and there are currently no universally acceptable criteria for its diagnosis $(31,32)$. Treatment of VCI is becoming increasingly important in countries with aging populations, as early treatment could prevent or slow the rate of progression from mild cognitive impairment to vascular dementia. Therefore, there has been an increase in research aimed at developing effective drugs for the treatment of VCI.

3-N-Butylphthalide (NBP) has been shown to be effective not only in protecting against ischemic cerebral injury, but also in ameliorating VCI in patients with dementia (33). The underlying mechanisms include enhancement of antioxidation, improvement of mitochondrial dysfunction, inhibition of neuronal apoptosis and autophagy, reduction of endoplasmic reticulum stress and upregulation of the Sonic hedgehog/Protein patched homolog 1 pathway (34-36). However, further studies are needed to determine if the neuroprotective effects of NBP in VCI are correlated with the SIRT1/BDNF pathway. The present study investigated the therapeutic efficacy of NBP against ischemic injury in VCI rats and examined the modulatory effect of NBP on the SIRT1/BDNF signaling pathway. 


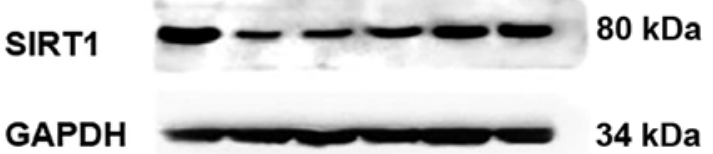

C $M \quad S+N$ B $+N$ L-N H-N

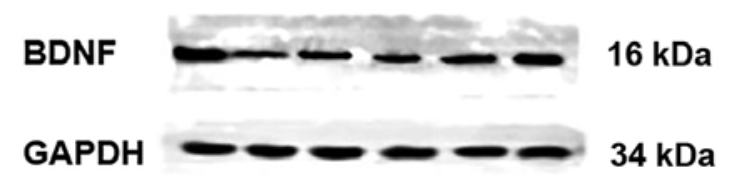

C M S+N B+N L-N H-N
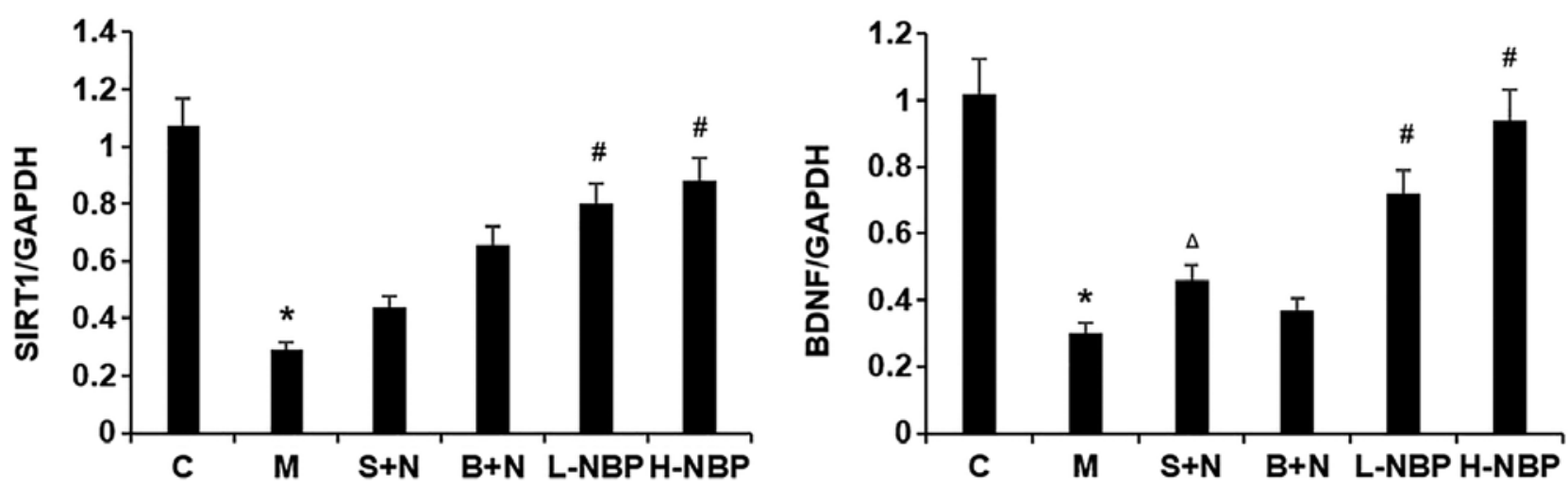

Figure 6. Effects of NBP on the expression levels of SIRT1 and BDNF in the hippocampus of vascular cognitive impaired rats by western blotting. NBP increased SIRT1 and BDNF protein expression levels. BDNF expression level was reduced in the S+N group, but SIRT1 expression was not decreased in the $\mathrm{B}+\mathrm{N}$ group, compared with the $\mathrm{H}-\mathrm{NBP}$ group. Data are presented as the mean $\pm \mathrm{SD} .{ }^{*} \mathrm{P}<0.05$ vs. $\mathrm{C}$ group. ${ }^{\#} \mathrm{P}<0.05$ vs. $\mathrm{M}$ group. ${ }^{\Delta} \mathrm{P}<0.05$ vs. $\mathrm{H}-\mathrm{NBP}$ group. $\mathrm{NBP}$, 3-N-butylphthalide; BDNF, brain-derived neurotrophic factor; SIRT1, silent information regulator 1; H-NBP, high dose NBP group (60 mg/kg); L-NBP, low dose NBP group (30 mg/kg); S+N, SIRT1 inhibitor + NBP (60 mg/kg) group; B+N, BDNF inhibitor + NBP (60 mg/kg); C, control; M, model group.

BCCAO has been widely used to induce ischemic injury in VCI research (37). The present results suggested that NBP may protect against ischemic injury-induced learning and memory impairment in both T-maze and MWM tests in a BCCAO model of VCI in rats. The present results also indicated that the neuroprotective effect of NBP may involve the SIRT1-BDNF signaling pathway. Furthermore, NBP also increased downstream BDNF expression levels by ameliorating upstream SIRT1 expression in the hippocampus of VCI rats.

Silent information regulator 1 (SIRT1) is closely related to aging, and is expressed in the liver, skeletal muscle and brain (38). SIRT1 brain levels are notably higher compared with levels in other tissues in mammals $(39,40)$, especially in the hippocampus, which is closely associated with learning and memory (41). SIRT1 has been shown to affect complex biological processes, including cognitive decline $(42,43)$. The present results suggested that ischemic injury induced learning and memory decline as well as decreasing SIRT1 expression levels in rats, and this process could be reversed by NBP treatment. Collectively, the present results suggested that the neuroprotective effect of NBP may be partially via the upregulation of SIRT1.

BDNF is a neuroprotective factor that is widely distributed in the central nervous system and plays a significant role in neurodegenerative diseases (44). BDNF binds to its receptor TrkB and activates the downstream signaling pathways to regulate synaptic function and maintain neuronal survival $(45,46)$. BDNF has been demonstrated to exert substantial neuroprotective effects and to improve neurological deficits (47). The present results suggested that cognitive impairment, as determined by T-maze and MWM tests, was associated with decreased BDNF expression levels compared with control rats, thus suggesting that BDNF was downregulated after ischemic injury. However, this downregulation of BDNF was reversed by NBP treatment.
Numerous studies have reported that NBP is a multi-target drug and its multiple mechanisms of action is effective in numerous conditions, such as Alzheimer's disease, Parkinson's disease and demyelination diseases (48-50). Previous studies revealed that NBP could support rebuilding of the collateral circulation, and had antithrombotic, anti-apoptotic and antioxidant effects, coupled with protection of mitochondrial functions $(5,51,52)$. The present study investigated the SIRT1/BDNF signaling pathway in rats with ischemic damage, and examined the relationship between NBP treatment and this signaling pathway. To the best of our knowledge, few studies have assessed the effect of NBP on VCI via the SIRT1/BDNF signaling pathway. Zeng and Yang (53) reported that SIRT1 may protect retinal neurons and visual function via the regulation of the BDNF/TrkB signaling pathway. In addition, Jiang et al (54) reported that SIRT1 had a neuroprotective effect in patients with Huntington's disease, which was associated with upregulation of the BDNF/TrkB signaling pathway. The present results suggested that NBP could significantly reduce the escape latency and swimming distance, increase the time in the targeted quadrant and increase the expression levels of SIRT1 and BDNF in VCI rats. In addition, the present results suggested that NBP improved spatial learning in VCI rats via activating the SIRT1/BDNF pathway. Injection of a SIRT1 inhibitor prevented the NBP-induced improvement in cognition and the elevation of BDNF expression levels, while injection of a BDNF inhibitor could not reverse the process. Therefore, the present results indicated that NBP increased SIRT1 expression level, which activated the downstream signaling pathway molecule BDNF, and resulted in partial recovery of some cognitive functions in VCI rats.

In conclusion, NBP may improve the recovery of cognitive function in rats after cerebral ischemic injury via upregulation of the SIRT1/BDNF signaling pathway. The SIRT1 may be 
the upstream regulatory element promoting the expression of BDNF and thereby improving cognitive deficits in VCI rats.

\section{Acknowledgements}

Not applicable.

\section{Funding}

The current study was supported by National Key R\&D Program of China (grant no. 2018YFC1311600), Liaoning Province Nature Science Foundation of China (grant no. 2015020471) and Science and Technology Program of Shenyang (grant no. 17-230-930).

\section{Availability of data and materials}

The datasets used and/or analyzed during the present study are available from the corresponding author on reasonable request.

\section{Authors' contributions}

RZ designed the study. WL established the rat models. QZ and HL collected and analyzed all experimental data. AT and WL drafted the manuscript and contributed substantially to its revision. AT performed the statistical analysis. All authors read and approved the final manuscript.

\section{Ethics approval and consent to participate}

Experiments were approved by The China Medical University Animal Care and Use Committee, and adhered to The Chinese Academy of Science Guidelines for Care and Use of Laboratory Animals.

\section{Patient consent for publication}

Not applicable.

\section{Competing interests}

The authors declare that they have no competing interests.

\section{References}

1. Du J, Ma M, Zhao Q, Fang L, Chang J, Wang Y, Fei R and Song X: Mitochondrial bioenergetic deficits in the hippocampi of rats with chronic ischemia-induced vascular dementia. Neuroscience 231: 345-352, 2013.

2. Akinyemi R, Mukaetova-Ladinska E, Attems J, Ihara M and Kalaria RN: Vascular risk factors and neurodegeneration in ageing related dementias: Alzheimer's disease and vascular dementia. Curr Alzheimer Res 10: 642-653, 2013.

3. Benisty S: Current concepts in vascular dementia. Geriatr Psychol Neuropsychiatr Vieil 11: 171-180, 2013 (In French).

4. GrinbergLT,NitriniR,SuemotoCK,LucenaFerretti-RebustiniRE, Leite RE, Farfel JM, Santos E, Andrade MP, Alho AT, Lima Mdo C, et al: Prevalence of dementia subtypes in a developing country: A clinicopathological study. Clinics (Sao Paulo) 68: 1140-1145, 2013.

5. Qi Q, Xu J, Lv P, Dong Y, Liu Z, Hu M, Xiao Y, Jia Y, Jin W, Fan M, et al: DL-3-n-butylphthalide alleviates vascular cognitive impairment induced by chronic cerebral hypoperfusion by activating the Akt/Nrf2 signaling pathway in the hippocampus of rats. Neurosci Lett 672: 59-64, 2018.
6. Han QY, Zhang H, Zhang X, He DS, Wang SW, Cao X, Dai YT, $\mathrm{Xu}$ Y and Han LJ: dl-3-n-butylphthalide preserves white matter integrity and alleviates cognitive impairment in mice with chronic cerebral hypoperfusion. CNS Neurosci Ther 25:1042-1053, 2019.

7. North BJ and Verdin E: Sirtuins: Sir2-related NAD-dependent protein deacetylases. Genome Biol 5: 224, 2004.

8. Seo JS, Moon MH, Jeong JK, Seol JW, Lee YJ, Park BH and Park SY: SIRT1, a histone deacetylase, regulates prion protein-induced neuronal cell death. Neurobiol Aging 33: 1110-1120, 2012.

9. Zu G, Ji A, Zhou T and Che N: Clinicopathological significance of SIRT1 expression in colorectal cancer: A systematic review and meta analysis. Int J Surg 26: 32-37, 2016.

10. Wang Y, Liang Y and Vanhoutte PM: SIRT1 and AMPK in regulating mammalian senescence: A critical review and a working model. FEBS Lett 585: 986-994, 2011.

11. Yang Y, Duan W, Li Y, Yan J, Yi W, Liang Z, Wang N, Yi D and Jin Z: New role of silent information regulator 1 in cerebral ischemia. Neurobiol Aging 34: 2879-2888, 2013.

12. Xia Y, Wang CZ, Liu J, Anastasio NC and Johnson KM: Brain-derived neurotrophic factor prevents phencyclidine-induced apoptosis in developing brain by parallel activation of both the ERK and PI-3K/Akt pathways. Neuropharmacology 58: 330-336, 2010.

13. Castillo DV and Escobar ML: A role for MAPK and PI-3K signaling pathways in brain-derived neurotrophic factor modification of conditioned taste aversion retention. Behav Brain Res 217: 248-252, 2011.

14. Pandey SC, Zhang H, Roy A and Misra K: Central and medial amygdaloid brain-derived neurotrophic factor signaling plays a critical role in alcohol-drinking and anxiety-like behaviors. J Neurosci 26: 8320-8331, 2006.

15. Kramár EA, Chen LY, Lauterborn JC, Simmons DA, Gall CM and Lynch G: BDNF upregulation rescues synaptic plasticity in middle-aged ovariectomized rats. Neurobiol Aging 33: 708-719, 2012.

16. Vetrovoŭ OV, Rybnikova TS and Samoŭlov MO: Effect of hypoxic postconditioning on the expression of antiapoptotic protein Bcl-2 and neurotrophin BDNF in CA1 hippocampal field of rats surviving severe hypoxia. Morfologiia 145: 16-20, 2014 (In Russian).

17. Zhang T, Yan W, Li Q, Fu J, Liu K, Jia W, Sun X and Liu X: 3-n-Butylphthalide (NBP) attenuated neuronal autophagy and amyloid- $\beta$ expression in diabetic mice subjected to brain ischemia. Neurol Res 33: 396-404, 2011.

18. Peng Y, Xu SF, Wang L, Feng YP and Wang XL: Effects of chiral $\mathrm{NBP}$ on cerebral infarct volume due to transient focal cerebral ischemia. Zhongguo Xinyao Zazhi 14: 420-423, 2005.

19. Dong GX and Feng YP: Effects of 3-N-butylphthalide on cortical calcineurin and calpain activities in focal cerebral ischemia rats. Yao Xue Xue Bao 35: 790-792, 2000 (In Chinese).

20. Liu CL, Liao SJ, Zeng JS, Lin JW, Li CX, Xie LC, Shi XG and Huang RX: Dl-3n-Butylphthalide prevents stroke via improvement of cerebral microvessels in RHRSP. J Neurol Sci 260: 106-113, 2007.

21. Peng Y, Hu Y, Xu S, Feng N, Wang L and Wang XL: L-3-n-butylphthalide regulates amyloid precursor protein processing by PKC and MAPK pathways in SK-N-SH cells over-expressing wild type human APP695. Neurosci Lett 487: 211-216, 2011.

22. Feng X, Peng Y, Liu M and Cui L: DL-3-n-butylphthalide extends survival by attenuating glial activation in a mouse model of amyotrophic lateral sclerosis. Neuropharmacology 62: 1004-1010, 2012.

23. Peng Y, Xu S, Chen G, Wang L, Feng Y and Wang X: 1-3-n-Butylphthalide improves cognitive impairment induced by chronic cerebral hypoperfusion in rats. J Pharmacol Exp Ther 321: 902-910, 2007.

24. Ghanbarabadi M, Iranshahi M, Amoueian S, Mehri S, Motamedshariaty VS and Mohajeri SA: Neuroprotective and memory enhancing effects of auraptene in a rat model of vascular dementia: Experimental study and histopathological evaluation. Neurosci Lett 623: 13-21, 2016.

25. Lee $\mathrm{CH}$, Park JH, Ahn JH and Won MH: Effects of melatonin on cognitive impairment and hippocampal neuronal damage in a rat model of chronic cerebral hypoperfusion. Exp Ther Med 11: 2240-2246, 2016.

26. Deacon RM and Rawlins JN: T-maze alternation in the rodent. Nat Protoc 1: 7-12, 2006.

27. Vorhees CV and Williams MT: Morris water maze: Procedures for assessing spatial and related forms of learning and memory. Nat Protoc 1: 848-858, 2006. 
28. Bowler JV: The concept of vascular cognitive impairment J Neurol Sci 203-204: 11-15, 2002.

29. Bowler JV: Modern concept of vascular cognitive impairment. Br Med Bull 83: 291-305, 2007.

30. Gorelick PB, Scuteri A, Black SE, Decarli C, Greenberg SM, Iadecola C, Launer LJ, Laurent S, Lopez OL, Nyenhuis D, et al: Vascular contributions to cognitive impairment and dementia: A statement for healthcare professionals from the American heart association/American stroke association. Stroke 42: 2672-2713, 2011.

31. Román GC, Sachdev P, Royall DR, Bullock RA, Orgogozo JM, López-Pousa S, Arizaga R and Wallin A: Vascular cognitive disorder: A new diagnostic category updating vascular cognitive impairment and vascular dementia. Neurol Sci 226: 81-87, 2004.

32. O'Brien JT: Vascular cognitive impairment. Am J Geriatr Psychiatry 14: 724-733, 2006.

33. Jia J, Wei C, Liang J, Zhou A, Zuo X, Song H, Wu L, Chen X, Chen S, Zhang J, et al: The effects of DL-3-n-butylphthalide in patients with vascular cognitive impairment without dementia caused by subcortical ischemic small vessel disease: A multicentre, randomized, double-blind, placebo-controlled trial Alzheimers Dement 12: 89-99, 2016.

34. Chen N, Zhou Z, Li J, Li BC, Feng JH, He D, Luo Y, Zheng X, Luo $\mathrm{J}$ and Zhang J: 3-n-butylphthalide exerts neuroprotective effects by enhancing anti-oxidation and attenuating mitochondrial dysfunction in an in vitro model of ischemic stroke. Drug Des Devel Ther 12: 4261-4271, 2018.

35. Xu J, Huai Y, Meng N, Dong Y, Liu Z, Qi Q, Hu M, Fan M, Jin W and Lv P: L-3-n-Butylphthalide activates Akt/mTOR signaling, inhibits neuronal apoptosis and autophagy and improves cognitive impairment in mice with repeated cerebral ischemia-reperfusion injury. Neurochem Res 42: 2968-2981, 2017.

36. Niu XL, Jiang X, Xu GD, Zhang GM, Tang ZP, Yin N, Li XQ, Yang YY and Lv PY: DL-3-n-butylphthalide alleviates vascular cognitive impairment by regulating endoplasmic reticulum stress and the Shh/Ptch1 signaling-pathway in rats. J Cell Physiol 234 12604-12614, 2019.

37. Xing M, Sun Q, Wang Y, Cheng Y and Zhang N: Hydroxysafflor yellow A increases BDNF and NMDARs in the hippocampus in a vascular dementia rat model. Brain Res 1642: 419-425, 2016.

38. Ogawa T, Wakai C, Saito T, Murayama A, Mimura Y, Youfu S, Nakamachi T, Kuwagata M, Satoh K and Shioda S: Distribution of the longevity gene product, SIRT1, in developing mouse organs. Congenit Anom (Kyoto) 51: 70-79, 2011.

39. Michishita E, Park JY, Burneskis JM, Barrett JC and Horikawa I: Evolutionarily conserved and nonconserved cellular localizations and functions of human SIRT proteins. Mol Biol Cell 16 4623-4635, 2005.

40. Shan T, Wang Y, Wu T, Liu C, Guo J, Zhang Y, Liu J and Xu Z: Porcine sirtuin 1 gene clone, expression pattern, and regulation by resveratrol. J Anim Sci 87: 895-904, 2009.

41. Michán S, Li Y, Chou MH, Parrella E, Ge H, Long JM, Allard JS, Lewis K, Miller M, Xu W, et al: SIRT1 is essential for normal cognitive function and synaptic plasticity. J Neurosci 30: 9695-9707, 2010
42. Chen YY, Zhang L, Shi DL, Song XH, Shen YL, Zheng MZ and Wang LL: Resveratrol attenuates subacute systemic inflammation-induced spatial memory impairment via inhibition of astrocyte activation and enhancement of synaptophysin expression in the hippocampus. Ann Clin Lab Sci 47: 17-24, 2017.

43. Wang R, Zhang Y, Li J and Zhang C: Resveratrol ameliorates spatial learning memory impairment induced by $A \beta_{1-42}$ in rats. Neuroscience 344: 39-47, 2017.

44. Li XH, Chen C, Tu Y, Sun HT, Zhao ML, Cheng SX, Qu Y and Zhang S: Sirt1 promotes axonogenesis by deacetylation of Akt and inactivation of GSK3. Mol Neurobiol 48: 490-499, 2013.

45. Li XH, Lv BL, Xie JZ, Liu J, Zhou XW and Wang JZ: AGEs induce Alzheimer-like tau pathology and memory deficit via RAGE-mediated GSK-3 activation. Neurobiol Aging 33: 1400-1410, 2012

46. Watzlawick R, Howells DW and Schwab JM: Neuroprotection aftr traumatic brain injury. JAMA Neurol 73: 149-150, 2016.

47. Kaplan GB, Vasterling JJ and Vedak PC: Brain-derived neurotrophic factor in traumatic brain injury, post-traumatic stress disorder, and their comorbid conditions: Role in pathogenesis and treatment. Behav Pharmacol 21: 427-437, 2010.

48. Xiang J, Pan J, Chen F, Zheng L, Chen Y, Zhang S and Feng W: L-3-n-butylphthalide improves cognitive impairment of APP/PS1 mice by BDNF/TrkB/PI3K/AKT pathway. Int J Clin Exp Med 7: 1706-1713, 2014.

49. Koppula S, Kumar H, More SV, Kim BW, Kim IS and Choi DK: Recent advances on the neuroprotective potential of antioxidants in experimental models of Parkinson's disease. Int J Mol Sci 13: 10608-10629, 2012.

50. Wu Y, Huang Q, Liu X and Wei X: Dl-3-n-butylphthalide is effective for demyelination: A case-combined study. Clin Neurol Neurosurg 137: 83-88, 2015.

51. Zhang C, Zhao S, Zang Y, Gu F, Mao S, Feng S, Hu L and Zhang C: The efficacy and safety of Dl-3n-butylphthalide on progressive cerebral infarction: A randomized controlled STROBE study. Medicine (Baltimore) 96: e7257, 2017.

52. Wang X, Wang L, Sheng X, Huang Z, Li T, Zhang M, Xu J, Ji H, Yin J and Zhang Y: Design, synthesis and biological evaluation of hydrogen sulfide releasing derivatives of 3-n-butylphthalide as potential antiplatelet and antithrombotic agents. Org Biomol Chem 12: 5995-6004, 2014.

53. Zeng $\mathrm{Y}$ and Yang K: SIRTluin 1 participates in the process of age-related retinal degeneration. Biochem Biophys Res Commun 468: 167-172, 2015

54. Jiang M, Wang J, Fu J, Du L, Jeong H, West T, Xiang L, Peng Q, Hou Z, Cai H, et al: Neuroprotective role of Sirtl in mammalian models of Huntington's disease through activation of multiple Sirt1 targets. Nat Med 18: 153-158, 2011.

This work is licensed under a Creative Commons Attribution-NonCommercial-NoDerivatives 4.0 International (CC BY-NC-ND 4.0) License. 